\title{
Turin Shroud-like electric imaging connected to earthquakes
}

\author{
Giovanna de Liso ${ }^{1, a}$, Francesco Lattarulo ${ }^{2, b}$, and Giulio Fanti ${ }^{3, c}$ \\ ${ }^{1}$ Seismic Precursors Study Center, Pinerolo (Torino), Italy \\ ${ }^{2}$ Department of Electrical and Information Engineering, Technical University of Bari, Bari, Italy \\ ${ }^{3}$ Department of Industrial Engineering, University of Padova, Padova, Italy
}

\begin{abstract}
A large amount of long lasting experiments, carried out by the first author with the supervision of coauthoring experts in electrical imaging and Turin Shroud (TS) studies, was addressed to obtain TS-like impressions. To this end, the experiments was especially performed by using conducting objects previously enveloped in two-folded cloths, then inserted into a gap bounded by a pair of rocky plates. The prerequisite for image formation seems to be an especially abundant emission of radon, related to earthquakes, ultimately giving rise to a macro-scale background electric field of the order of $1 \mathrm{kV} / \mathrm{m}$ in the gap and, more importantly, to micro-scale field amplification. This additional field is localized in the air filled interstices of the exposed fabric, thus causing consequent aging. In spite of the complicate and unpredictable character of the natural phenomenology under examination, the investigators succeeded in categorizing different kinds of impressions and selecting one of them as being thoroughly successful. Specifically, the images of the selected category do exhibit basic physicochemical and optical features distinctive of the figure impressed on the Turin Shroud.
\end{abstract}

\section{Introduction}

The most important and in-depth scientific analysis of TS was performed in 1978 by STURP (Shroud of Turin Research Project) [1,2] although no explanations for the impressed human image were put forward [3-6]. Presently, the optical and physicochemical properties of the image result definitively assessed with the specification that they are not amenable to reproduction altogether, in spite of the efforts made to explain the debated imaging mechanism.

In agreement with [7], the authors are convinced that the best explanation for the TS imaging is based on electrostatics and that the impressive mechanism involves corona discharges (CD) [7-11]. To strengthen this statement, consider that a lot of experiments were carried out by the first author in a radon-invaded premise [12]. This investigator only adopted vegetal fabrics as covers. A collection of minute marks, overall appreciable at naked eye under form of indelible TS-like images of the enclosed bodies, was especially impressed on the inner side of the cover. It is worth noticing that the impressive

\footnotetext{
a e-mail: seismicprecursors@gmail.com

b e-mail: francesco.lattarulo@poliba.it

c e-mail: giulio.fanti@unipd.it
}

This is an Open Access article distributed under the terms of the Creative Commons Attribution License 4.0, which permits unrestricted use, distribution, and reproduction in any medium, provided the original work is properly cited. 
mechanism invariably manifested when seismic episodes occurred. The methodical character of the monitored phenomenon gives persuading arguments to sustain that imaging turned out to be a precursor of, because occurring some days before, earthquakes. However, differently from laboratory procedures in which the parameters of interest are usually subject to careful control, the physicochemical agents involved in this special class of natural events are to be considered quite unpredictable.

West Piedmont is a moderately seismic Italian region with radon-invaded areas especially located nearby Cuneo and Pinerolo. As a result of previous observations conducted in situ, a substantial correlation seems to hold between geological structure and abrupt changes of some data, specifically ambient radioactivity and geo-magnetism [12]. Just during these changes, images happened to be naturally impressed on the surface of the adopted vegetal fabrics, the latter covering conductors of different shape and material. This paper takes into account a reasoned selection, based on the facts reported in [5] and [6], of available experimental data for the postulated imaging mechanism, and its relevance to TS, to be better appreciated.

\section{Imaging hypothesis}

Owing to the special features of the resulting images, such as superficiality [4, 5], double superficiality [13] and color, the authors are inclined to claim the causal relationship between the imaging under examination and $\mathrm{CD}$, even though this phenomenon is triggered by an electrostatic mechanism still needing to be exhaustively ascertained and described. An indoor vertically oriented electric field, with strengths attaining the order of $1 \mathrm{kV} / \mathrm{m}$ in the gap between the plates where the object under test was positioned (see later for more detailed considerations), was monitored. This value, larger up to two orders of magnitude than one detected in the outer ambient, was reasonably due to the presence of the upper plate which partially obstructed the gas flow. As a consequence, the heavier nature of radon than pure air caused the former to laterally invade and accumulate into the gap, thus causing significant ionization increase, where the object was positioned. The first author observed that images are more easily formed if the inter-plate gap is almost four times the maximum height of the object as an example to the impressed image of a $1.8 \mathrm{~cm}$ thick snake, the two layers were distanced of $13 \mathrm{~cm}$.

The electric field is the result of ion separation reasonably ascribed to the different mobility of the positive and negative ion species subject to upward thrust. This is the case when sulphureous throws typically accompany the radon emission. A reasonable envision of the scenario pertaining to a radoninvaded natural cave is provided in [11]. In spite of the above supportive cause of field enhancement, the detected strength is apparently far below the expected CD onset level, which implies that some overlooked additional mechanism could have a crucial part to play. This is the case, in the authors' opinion, whenever a large amount of gas filled interstices - as those characterizing porous materials (i.e., cardboard and fabrics), sometimes placed in intimate contact with a conducting body, other times in isolation - is involved. As a result, an undetectable local amplification of the background electric field, by charge accumulation (space-charge distortion), takes place in the elemental interstices. Importantly consider that under normal ambient ionization, the often referred to as cutoff (or memory) effect due to small amounts of charge accumulation is only responsible for powerless pulsating partial discharges. These are substantially unable to impress the expected permanent marks on a dielectric barrier, even when the applied electric field is rather exceeding the $\mathrm{CD}$ onset level. On the other hand, provided the rate of ambient radioactivity is enhanced, the pulses easily tend to merge in time, thus causing a transition of the pulsative mode of the discharge into a glow-type one.

The transition could need rather moderate background electric fields, depending on the degree of ionization assumed for the ambient air. Under the described circumstances, proliferation of confined and severe discharges are overall capable to determine locally perceivable and stable physicochemical modifications. As a whole, these are termed accelerated aging of the exposed dielectric material. Parenthetically, the aging severity is rather the consequence of potently reacting chemical by-products 


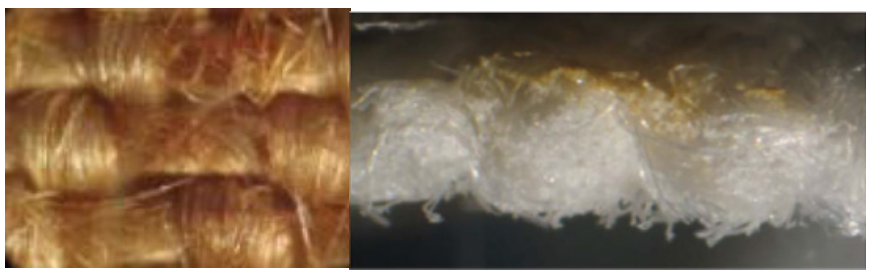

Figure 1. Image on a linen sample (left). Enlarged cross-section showing image superficiality (right).

of the discharges than of the direct, physical erosion of the latter in themselves (by ion bombardment). However, returning to the electrostatic phenomenon under examination, it is the interstitial amplification of the field, due to a large pre-ionization, that can account for the apparent decay of the CD onset level.

More precisely, the "macroscopic" notion of threshold applied to the CD triggering is even inappropriate in itself under the described conditions, because a nonzero, non linearly growing current is already detectable when an initially vanishing exogenous electric field starts to rise (see again [11] and related references). This seems to be the case for the present investigation, in which electric currents considerably less than $200 \mu \mathrm{A}$ (peak value) and average strengths of $1 \mathrm{kV} / \mathrm{m}$, were monitored between the plates for periods of times limited to $200 \mathrm{~s}$. Presence of uranium is the prerequisite for radon effluxes; in fact, it was ascertained local presence of pechblenda and uranite rocks whose constituents are uranium phosphate and uranium oxide. Measured radioactivity at Torre Pellice usually ranges from 0.11 to $0.45 \mu \mathrm{S} / \mathrm{h}$, while outdoor and indoor radon attains peaks of $40 \mathrm{~Bq} / \mathrm{m} 3$ and $120 \mathrm{~Bq} / \mathrm{m} 3$ (data averaged over twelve hours). Radioactivity peaks of $57.0 \mu \mathrm{S} / \mathrm{h}$ and of $1500 \mathrm{~Bq} / \mathrm{m} 3$, related to special radon invasion in the ambient air, was observed during the experiments.

\section{Experimental apparatus and results}

All the experiments were performed in an underground cellar at Torre Pellice, where perturbations of environmental quantities related to seismic episodes was recorded. Specifically, presence of considerable rates of radon (measured radon concentration greater than $100 \mathrm{~Bq} / \mathrm{m} 3$ and radioactivity greater than $0.3 \mu \mathrm{S} / \mathrm{h}$ ) was estimated to be the prerequisite for successful experiments [12].

Linen, cotton and hemp clothes, covering up and down various objects, were put into the gap of the above-mentioned assembly made of gneiss plates. These are made by a pair of 3-cm thick gneiss layers of surface dimensions $2.0^{\prime} 0,5 \mathrm{~m}$ and mounted parallel to the ground with a gap ensured by conductive (iron or copper) or non-conductive (gneiss, marble, etc.) spacers.

The apparatus was placed in the neighborhoods of a fissure where considerable amounts of radon happen to come out especially before earthquakes. The clothes were soaked with different aqueous or oily solutions (aloe, myrrh and balsam; dilution of aloe and myrrh not exceeding $20 \mathrm{~g} / \mathrm{l}$ ) ensuring wetting for as long as imaging developed. Objects of different nature (vegetal, animal, mineral, synthetic) were covered by the cloth for a period of time up to 48 hours. Images deriving from successful tests appear superficial, see Fig. 1, and often visible on both sides of the cloth when insulating spacers was adopted (namely, provided the electric field just surrounding the enveloped bodies under test was uniform and vertical, or approximately so). No color penetrates the fabric thickness, so that the given double superficiality of the image turns out to be a further argument in favor of the corona discharge as direct agent. In fact, under specialized conditions, inner discharges are able to restart on the outer side of the cover as a result, say, of radon promoted photoionization. When this was the case, the inner side of the cloth, the one in contact with the object, however exhibited more intense and resolved images than the outer side. 




Figure 2. Image examples of conducting objects, notably a snake and a key (upper). Detailed reproduction of 3D features (lower).

According to Fig. 2, the images impressed on the inner side of the cover surprisingly manifest the $3 \mathrm{D}$ features of the covered conducting objects. This successful image transfer rather depends on the distribution of the electrostatic stress over the surface of the inner conducting object than on the way the conductor was tucked into the fabric. In this respect, consider that the exposure tests was in general performed after the upper piece of the folded fabric was simply placed on the object. Therefore, the albeit modest flexural stiffness of the adopted fabric was enough to accidentally facilitate some localized conductor/cover detachments.

Reiterate tests proved that the imaging activity could be deficient in those areas. It is often the case that these unintentional gaps are located precisely where the electrostatic stress decreases so much that the discharge activity turns off. This could be the case even neglecting, for the sake of simplicity, any concurrent barrier effect $[14,15]$. Therefore, special attention was paid to force every once in a while the cover, that is prone to freely drape by itself, to somewhat stand firm in intimate contact with the inner conductor. This has been done in order to prevent, as far as possible, that the detachments was somewhere responsible of unpredictable deficiencies of the electrostatic image transfer. As expected, indeed, the substantial result was that the electric field, intended as being nearly subtracted from the complex barrier effect, suffices to methodically cause undistorted imaging.

The 3D features appear transferred along with negativity, superficiality, double superficiality, resolution, non-contact effects, no fluorescence. Differently from alternative hypotheses on earthquake originated image formation - see, for example, the recently claimed neutron emission 
mechanism [16] - it can be said that only an electrostatics-based one is so far capable of explaining, as previously discussed, the ostensible body rendering impressed on the inner side of the unfolded cover. This performance, starting from an attractive analogy between irradiance and electrostatic field, has been discovered and carefully treated elsewhere [17] and later applied to a TS study [11].

To be more detailed, let a discharged and generically shaped conducting object is embedded in a uniform background electrostatic field. Also let a distanced observer is capable to "see" that fractional amount of the electric-field flux just as it is about, in some sense, to enter the facing side of the object surface. Therefore, the fictional observer could be led to rightfully understand that the described electrostatic scenario looks like an irradiance planar map, irrespective of the observer's line-of-sight. It is tacitly understood that the raised analogy applies to the same object regarded as being illuminated from afar by an incident plane wave's irradiance with the same orientation as that of the previous background electric field.

Of course, an equal amount of electric-field flow leaves the discharged conductor on its opposing side; however, this is not just of importance in the present context. Additionally, it is worth considering that the described observational feeling can in practice hold even if the actual 3D incidence surface is virtually laid flat after having been impressed during the exposure.

Parenthetically, it could be helpful for the reader to specify that the above referred to as incidence surface corresponds to the illuminated side of any given object. In other words, provided the shape of the covered body is a rather rounded off and/or moderately protruding one, an unimportant pattern disfiguration is merely perceived when the original 3D morphology of the incidence surface somehow happens to inelastically "spread over" a plane before being compared to the irradiance planar map.

This slight perception even more so applies to the physical case in which the inner side of a piece of fabric, used to cover the active conducting body, operates as a fitting material on which the recorded incidence pattern lies. The above activity applied to the inner conductor refers to the proliferation of surface discharges in turn triggered, and controlled in terms of severity, by the local electrostatic stress. Under the described circumstances, the disfiguration is detected within the uncertainties especially affecting this kind of investigations in which a common coverage is adopted. In fact, the lack of full adherence of the cover to the incidence surface and existence of a given threshold effect for the aging process to develop on the facing cover, turn out to be in-tandem drawbacks for image changes to be substantially undetectable. By the way, consider that a usual fabric can, broadly speaking, be categorized as a low-sensitive material when it is exposed to that rather gentle physicochemical threat represented by corona activity.

Figure 2 (lower panel) is the result of a shape-from-shading (SFS) code implemented for unambiguously reconstructing the 3D features of the objects whose impressed images are visible on the upper panel. Note that these objects are shaped according to the specialized conditions remarked above for the disfiguration to become indiscernible. Parenthetically, Fig. 2 (lower) does deserve special appreciation since SFS is usually identified in computer vision as a demanding inverse problem because of the general shape of the object to be recovered [18].

In theory, such a hard problem is rigorously well posed only when a restricted number of canonical configurations are treated. However, reliable numerical strategies, often based on iterative methods, are currently adopted to circumvent the raised formal disadvantage and safely reconstruct general objects. An additional difficulty arises when the shading of the starting planar pattern looks, this is the case, rather tenuous, disturbed and with faded contours.

An environmental scanning by an electron microscope (ESEM, model FEI Quanta 200) and a X-ray fluorescence spectroscopy (energy dispersive X-ray spectroscopy, EDS or EDX) was adopted together with the intention of carefully detecting the presence, if anything, of deposited foreign material and their properties. No deposition is methodically evidenced on the individual threads, as the enlargement reproduced in Fig. 3 shows, for TS-like images. In other words, the image category conversely displaying microscopic properties with ostensible departure from the TS ones, are also polluted. 


\section{SHS Web of Conferences}

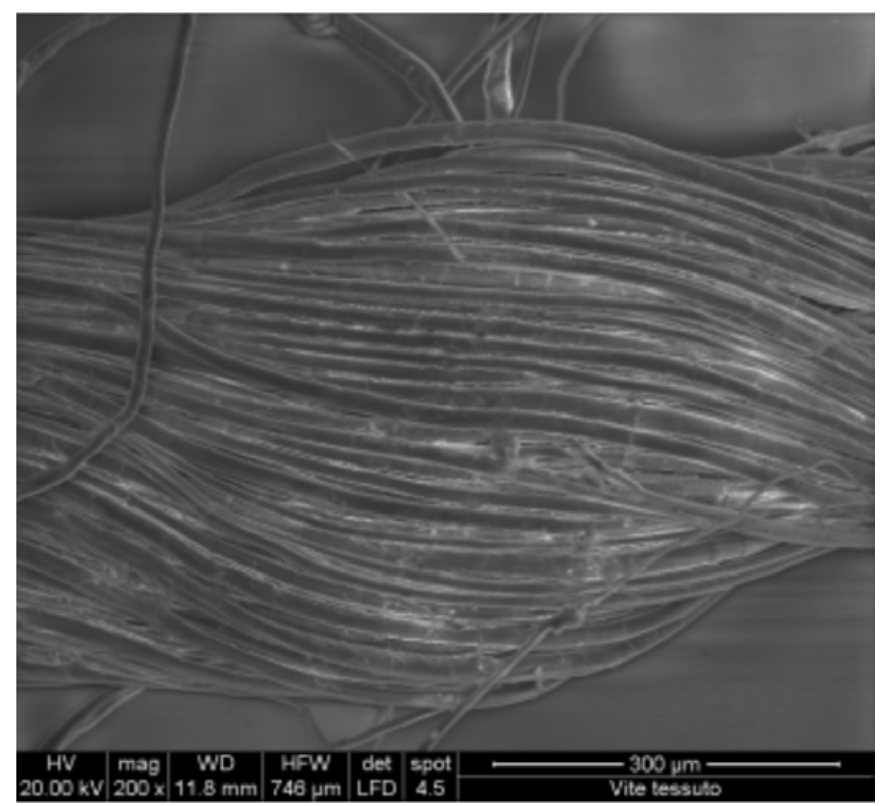

Figure 3. Colored threads revealing the lack of foreign material.

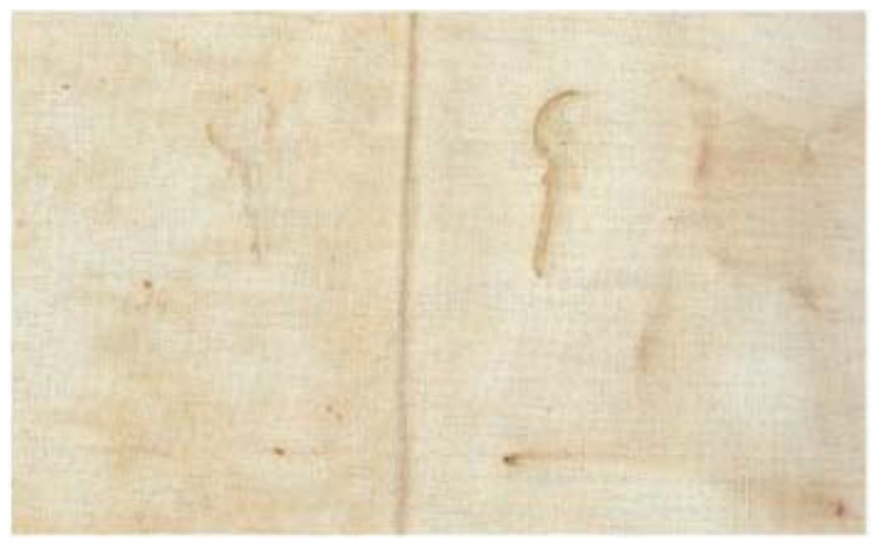

Figure 4. Frontal and dorsal images of a key on a unfolded and planar cover made of linen. The symmetrical image has been impressed on the inner side, placed in intimate contact with the key, of the folded linen. The given overall image is the result of an exposition to a transversally oriented exogenous electric field of the order of $1 \mathrm{kV} / \mathrm{m}$.

This is a consequence of projection of foreign material from the more stressed parts of the facing conductor under corona. A detailed description of these departed results, as one reported in [12], is intended to give further evidence on the difficulties that arise in trying to meet carefully the micro-scale conditions in conjunction with macro-scale ones for a satisfactory replica of TS-like images, ever more so in a natural way. An example of successful pair of images is reproduced in Fig. 4, where the inner side of the folded cover is visible.

This pair of superficial impressions of the same conductor is macroscopically characterized, in particular, by rather uniformly distributed (slight contrast) dark-yellow color, a prominent detail better 
appreciable by the enlargement of Fig. 1. Because of the lack of pigment, this result is, even by elimination, accounted for in terms of the discussed chemical reaction of the fibers as a consequence of the discharges. The color is visible only after three hours, with the specification that the ambient humidity is not exceeding $40 \%$. In fact, large percentages of water molecules in the gaseous filler is detrimental for an electric-field related effect to manifest.

\section{Conclusion}

What matters to succinctly highlight is that:

- the furnished images appear to be originated by earthquakes, with best results given about one day before individual seismic events;

- the formation mechanism could be connected to high rates of radon ultimately promoting corona activity on the surface of conductors placed in radon-prone ambient;

- the micro-scale component of the electric field, due to proliferation of an exceedingly large accumulation of interstitial charge, is deduced to be the first-order intensification mechanism, hence, macroscopically interpreted in terms of abatement for the $\mathrm{CD}$ onset level under pre-ionized conditions;

- on the other hand, a second-order additional intensification of the macro-scale electric field could admissibly be ascribed to the simultaneous magnetostrictive and piezoelectric performances of the implied gneiss;

- the competing electrostatics rather applied to the Earth's surface charge layer, thus connected to undetectable activation of p-holes [19,20], is deliberately cast aside against the present one that takes into account the detectable radon agent and related convincing arguments;

- best quality images are impressed on cloths previously soaked in salt water;

- regardless of handling and preparation of the fabric samples to be tested, the key answer to the rationale behind a successful object rendering lies with the claimed analogy between electric field and irradiance.

All things considered, and in agreement with [7] and [17], the experimental data provided by this investigation can of course only give rise to a speculative description of the TS figure. That is, the examined imaging should for the present be understood as a contribution to the current discussion about the origin of the human figure impressed on the TS. Notwithstanding this, the consistency in argument is sustained by meaningful observables pushing further to vindicate partial discharges as a reasonable candidate to the image formation examined in this paper.

\section{References}

[1] E.J. Jumper et al. (6 authors in all), "A comprehensive examination of the various stains and images on the Shroud of Turin", ACS Advances in Chemistry, Archaeological Chemistry III, vol. 205, pp. 447-476, 1984

[2] L.A. Schwalbe and R.N. Rogers, "Physics and chemistry of the Shroud of Turin, a summary of the 1978 investigation", Analytica Chimica Acta, vol. 135, pp. 3-49, 1982

[3] R. Basso and G. Fanti, "Optics Research Applied to the Turin Shroud: Past, Present and Future" in Optics Research Trends, P.V. Gallico Ed., New York: Nova Science, 2007, p. 4

[4] G. Fanti, J.A. Botella, P. Di Lazzaro, T. Heimburger, R. Schneider and N. Svensson "Microscopic and Macroscopic Characteristics of the Shroud of Turin Image Superficiality", J. of Imaging Sci. Technol., vol. 54, p. 040201-1/8, 2010

[5] Fanti G., J.A. Botella, F. Crosilla, F. Lattarulo, N. Svensson, R. Schneider, A.D. Whanger, "List of Evidences of the Turin Shroud, Int. Workshop on the Scientific Approach to the Acheiropoietos 
Images", ENEA Research Center of Frascati (Italy), 2012, ISBN 978-88-8286-232-9, 2010, http://www . acheiropoietos.info/proceedings/FantiListWeb.pdf

[6] G. Fanti et al. (24 authors): "Evidences for Testing Hypotheses about the Body Image Formation of The Turin Shroud", III Dallas International Conference on the Shroud of Turin: Dallas, Texas, September 8-11, 2005, http://www.dim.unipd.it/fanti/docLIST.pdf; http://www.shroud.com/pdfs/doclist.pdf

[7] G. Fanti, "Hypotheses regarding the formation of the body image on the Turin Shroud. A critical compendium", J. of Imaging Sci. Technol., vol. 55, p. 060507, 2011

[8] G. Fanti, "Can Corona Discharge explain the body image formation of the Turin Shroud?", J. of Imaging Science and Technology, vol. 54, pp. 020508-1/10, 2010

[9] F. Lattarulo, "TS image explained by a sismo-electric process" (translated from Italian). III Congresso internazionale di studi sulla Sindone, Torino, June 5-7 1998, pp. 334-346

[10] G. Fanti, F. Lattarulo, O. Scheuermann: "Body Image Formation Hypotheses Based on Corona Discharge", the Third Dallas International Conference on the Shroud of Turin: Dallas, Texas, September 8-11, 2005, http://www.dim.unipd.it/fanti/corona.pdf

[11] V. Amoruso and F. Lattarulo, "A physicochemical interpretation of the Turin Shroud imaging", Scientific Research and Essays, vol. 7, n. 29, July 2012, pp. 2554-2579

[12] G. de Liso, "Shroud-like experimental image formation during seismic activity", ENEA Research Center of Frascati (Italy), ISBN 978-88-8286-232-9, 2010, http://www.acheiropoietos . info/proceedings/DeLisoWeb.pdf

[13] G. Fanti and R. Maggiolo, "The double superficiality of the frontal image of the Turin Shroud", J. of Optics A: Pure and Applied Optics, vol. 6, pp. 491-503, 2004

[14] M. Abdel-Salam, H. Singer and A. Ahmed, "The barrier effect on the onset voltage of negative corona in air", J. Phys D: Appl. Phys. vol. 30, pp. 1017-1028, 1997

[15] F.V. Topalis and M.G. Danikas, "Breakdown in air gaps with solid insulating barrier under impulse voltage stress". Facta Universitatis (NIŠ), vol. 18, pp. 97-104, 2005

[16] A. Carpinteri, G. Lacidogna and O. Borla, "Is the Shroud of Turin in relation to the Old Jerusalem historical earthquake?" Meccanica, 2014, DOI: 10.1007/s11012-013-9865-x, http://www. aimetatorino2013.it/cdrom/cdrom_pdf_fullpaper/003510032801.pdf

[17] F. Lattarulo and V. Amoruso, "Electromagnetic treatment for the inverse reflectance-based shape recovery problem”. Int. J. Appl. Electromagnetics Mech., vol. 37, pp. 231-240, 2011

[18] R. Klette, R. Kozera and K. Schlüns, "Reflectance-Based Shape Recovery", in Handbook of Computer Vision and Applications, B. Jähne, H. Haußecker and P. Geißler editors, vol. 2 (Signal processing and Pattern Recognition), ch. 19, Acad. Press, San Diego, 1999

[19] F.T. Freund, "Rocks that crackle and sparkle and glow - Strange pre-earthquake phenomena - J. Sci. Exploration, vol. 17, pp. 37-71, 2003

[20] Robert Thériault, France St-Laurent, Friedemann T. Freund, and John S. Derr, "Prevalence of Earthquake Lights Associated with Rift Environments", Seismological Res. Letters, vol. 85, pp. 159-178, 2014 